\title{
Role of 25-Hydroxy Vitamin D Level in Ischemic Stroke Patients
}

\author{
Authors \\ Arvind Kumar*, Prem Singh**1, Gaurav Gupta***, Brijesh Kumar*, \\ Mahendra Singh**2 ${ }^{2}$ S.K. Gautam*, Tanu Midha*³ Anjana Sankhwar*4 \\ *Associate Professor of Medicine ${ }^{*}$ Associate Professor of SPM ${ }^{*}$ Medical officer in PMS \\ $*^{*}{ }^{1}$ Professor of Medicine \\ **2 Professor and Head of Pathology ***Junior Resident of Medicine \\ GSVM Medical College, Kanpur \\ Corresponding Author
}

\section{Gaurav Gupta}

\begin{abstract}
Background: Stroke is one of the most frequent causes of death and disability worldwide with a big significant clinical and socioeconomic impact. It has a heterogeneous etiology including unmodifiable risk factors like genetic, age, and sex, and modifiable risk factors including hypertension, diabetes mellitus, dyslipidemia, and smoking. In the recent past, studies have indicated that vitamin D deficiency is predictive for future strokes, but the evidence is limited.
\end{abstract}

Aims and Objective: To determine the role of 25-hydroxyvitamin D levels on the occurrence of ischemic stroke and its comparison with age and sex-matched controls.

Material and Methods: A case-control study was done and a total of 48 patients with acute ischemic stroke were chosen. An equal 48 controls were included in this study for the comparison of outcome. 25 hydroxy vit D levels were measured by chemiluminescence assay using commercially available kits.

Results: Mean Vitamin D Levels among cases and controls were $15.46 \pm 8.41$ and $26.74 \pm 13.28$, respectively. There is a significant difference observed between the case and control group while comparing mean vitamin D levels, p-value $<0.0001$ (highly significant). Vitamin D deficiency ( $<20 \mathrm{ng} / \mathrm{ml}$ ) is inversely related to the incidence of ischemic stroke (odds ratio $=4.109$ within a $95 \%$ confidence interval of 1.74 to 9.71 ).

Conclusion: We found that vitamin D deficiency is associated with an increased risk of ischaemic stroke.

Keywords: stroke, 25-hydroxy vitamin D.

\section{Introduction}

Stroke is sudden onset of focal neurological deficit, one of the major causes of morbidity and mortality. Stroke leads to $1 \%$ of mortality among hospital deaths in India, also accounts for $4 \%$ of admissions in Medical wards and almost $20 \%$ of all patients admitted with neurologic disorder. ${ }^{1}$

The WHO has defined stroke or cerebrovascular disease as a syndrome of rapidly eveloping clinical symptoms, with an apparent vascular cause, of focal or global disturbance of cerebral function lasting for $\geq 24$ hours or leading to death. ${ }^{2}$ Stroke has a heterogeneous etiology including unmodifiable risk factors like genetic, age and sex, and modifiable risk factors including hypertension, diabetes mellitus, dyslipidemia, sedentary lifestyle, obesity, smoking. ${ }^{3}$ Yet, risk factors aren't identified in a sizable amount of 
cases with stroke. Hence, plenty of epidemiological studies are being done to spot novel emerging risk factors and their role in reducing the incidence of stroke and their exact nature of association with stroke. ${ }^{5}$ In the recent past, researchers have given much emphasis to one such risk factor i.e. vitamin D deficiency. Studies have indicated that low vitamin D status is predictive for future strokes, but the evidence is limited. ${ }^{6-8}$

Vitamin D is a 9,10-secosteroid and one of the lipid-soluble vitamins and the most common forms in humans are vitamin D3 (cholecalciferol) and Vitamin D2 (ergocalciferol) ${ }^{10}$. Vitamin D3 is synthesized from photoisomerization of 7dehydrocholesterol within the skin's epidermal layer in the presence of ultraviolet light. Then, undergo two temperature dependent hydroxylation steps: first inactive vitamin $\mathrm{D}$ is hydroxylated in the liver to make 25-hydroxyvitamin $\mathrm{D}$, then classically within the kidney by $1-\alpha$-hydroxylase to become biologically active form 1,25dihydroxyvitamin D3. The second hydroxylation step may also occur in macrophages, $\mathrm{T}$ cells and neurons. ${ }^{10}$ Vitamin D2 is a plant sterol found in foods, like fatty fish, cod-liver oil and eggs. Vitamin $D$ is crucial for the human body to maintain a balance between calcium and phosphorus. Inadequate vitamin D can cause weakness, reduced bone mineralization, increased bone loss and fractures. ${ }^{9} 25$ hydroxyvitamin $\mathrm{D}$ is a marker of vitamin D status in the human body.20 Vitamin $D$ is measured by levels of a metabolically inactive precursor, 25hydroxyvitamin D3 [25(OH)D3], since the serum concentration of $1,25(\mathrm{OH}) 2 \mathrm{D} 3(\leq 100 \mathrm{pM})$ is significantly lower than $25(\mathrm{OH}) \mathrm{D} 3(\leq 100 \mathrm{nM})$, also the half-life of $1,25(\mathrm{OH}) 2 \mathrm{D} 3$ is incredibly less around 15 hours as compared to $25(\mathrm{OH}) 2 \mathrm{D} 3$ that has a circulatory lifetime of around 2 to 3 weeks. The very fact that levels of $1,25(\mathrm{OH}) 2 \mathrm{D} 3$ are easily influenced by PTH, calcium, phosphorus, make it a least preferred marker for vitamin D status of the body. ${ }^{11}$
Vitamin D deficiency is pandemic, yet it is the most under-diagnosed and under-treated nutritional deficiency in the world. ${ }^{12-14}$ In addition to its well-accepted role as a major regulator of calcium and bone metabolism, in recent years, it has received attention for its non-skeletal effects, its deficiency has been related to numerous health outcomes including several cardiovascular risk factors, including hypertension ${ }^{15}$, insulin resistance $^{16}$, cerebral small vessel disease ${ }^{17}$, stroke severity ${ }^{18}$, infarct volume ${ }^{19}, \operatorname{mood}^{20}$ and $\operatorname{cognition}^{20}$ in previous studies. However, studies considering these interactions in logistic regression models are very few in numbers.

Vitamin D is also neuroprotective because of its involvement in regulating the release of neurotrophic factors and in maintaining the bloodbrain barrier integrity. ${ }^{21}$ It has been linked to vasoprotective potential including slowing down of atherosclerosis, promotion of endothelial function, suppression of renin-angiotensin-aldosterone system thereby reduction of the risk of hypertension. ${ }^{21}$ Moreover, deficiency of vitamin D influences vascular remodelling through modulation of smooth muscle cell proliferation, inflammation and thrombosis. These vascular changes can eventually cause a stroke. ${ }^{22}$

Asians, notably Indians, are reported to be extremely vitamin D deficient with the lowest serum 25-hydroxyvitamin D $(25(\mathrm{OH}) \mathrm{D})$ levels amongst other ethnicities. ${ }^{23-25}$ Studies from India have uniformly pointed to have low $25(\mathrm{OH}) \mathrm{D}$ levels within the Indian population in all age groups and all regions, despite lots of sunshine. ${ }^{23,24}$ India, with a population size of 1.3 billion and co-occurring epidemics of vitamin D deficiency and stroke, ${ }^{12-14}$ provides a robust platform for assessment of the interrelationship between vitamin D status and risk of stroke. Reported clinical studies indicate an association of vitamin D status and stroke; but, most of these results are based on populations of Caucasian descent. $^{26-28}$ Generalization or extrapolation of results of association studies across various populations might not be reasonable. ${ }^{29}$ Hence, 
independent assessments of the impact of vitamin D standing on the risk of stroke across different subpopulations become necessary. Given these discrepancies, we undertook the current study to assess the association between vitamin D status and risk of ischemic stroke based on a population from Uttar Pradesh, a northern state of India.

\section{Aims and Objectives}

To determine the role of 25-hydroxyvitamin D levels on the occurrence of ischemic stroke and its comparison with age and sex-matched controls.

\section{Materials and Methods \\ Study Design}

We conducted a case control study over a period of one and half years (January 2019 to October 2020). The study was conducted at the department of internal medicine, GSVM Medical College Kanpur and subjects were recruited from Medicine Emergency and wards. The study was approved by the Ethics Committee of the institute ETHICS APPROVAL NUMBER (EC/BMHR/211/OCT/2020, dated 23/10/2020).

\section{Participants}

A total of 48 patients with acute ischemic stroke were chosen in the study. An equal 48 healthy controls were taken from normal healthy individuals attending medicine OPD/SEMIEMERGENCY. One case will be taken for each control for comparison of outcome. Sample size was calculated with reference to a pilot study done on 5 cases and 5 controls. They observed mean difference of vit D level was 4.7 in the study subjects. By using above reference values, Sample size was calculated by formula for calculating sample size for comparing 2 means at $95 \%$ confidence interval and $80 \%$ power, $5 \%$ level of significance. Sample size was calculated as 96 subjects with 48 subjects in both groups. Informed consent subjects with Age $>18$ years both genders with ischemic cerebrovascular accident identified based on clinical as well as laboratory and radiological evaluation (including $\mathrm{CT} / \mathrm{MRI}$ ) admitted in our hospital were included in our study. Subjects with previous history of TIA/CVA, on vitamin D and calcium supplementation, CKD, CLD, DIABETES MELLITUS, patients who underwent thrombolysis, cardio-embolic stroke patients were excluded from study.

\section{Procedure Methodology}

All the required details about cases such as demographic data (Age, gender, address, registration number, etc), clinical presentations (signs \& symptoms), general examination findings, systemic examination and Ischemic stroke findings were carried out. Blood sample was taken from all patients with acute stroke during 24 hour of admission to check 25-hydroxy vit $\mathrm{D}$ levels were measured by chemiluminescence assay using commercially available kits, fasting blood sugar, HbA1c, LFT, KFT and lipid profile. All patients underwent $\mathrm{CT}$ scan brain (plain) to establish the ischemic lesion if If patients had a normal CT scan brain, then ischemic stroke was diagnosed based on diffusion-weighted MRI. Age and sex matched healthy controls were selected and 25-hydroxy vit D levels were measured by chemiluminescence assay. All other biochemical measurements were performed as per the standard procedures.

\section{Statistical Analysis}

Data was analysed using Statistical Package of Social Sciences (SPSS, version 23.0) software and expressed as mean \pm standard deviation. Continuous data was analysed using student's Ttest. Inter group or intra group comparisons were done using independent sample test for comparison of mean values. Association between variables was considered statistically significant if p-value was $<0.01$.

\section{Observation and Result}

The mean age of the case group was $60.95 \pm 13.9$ years which is comparable [p value $=0.937$ (not significant)] to the mean age of the control group which was $61.16 \pm 11.6$ years. 
Table No -1 Average Age Between Cases and Controls

\begin{tabular}{|l|c|c|c|}
\hline & Case $(\mathrm{n}=48)$ & Control $(\mathrm{n}=48)$ & P value \\
\hline Mean Age (in years) & $60.95 \pm 13.9$ & $61.16 \pm 11.6$ & 0.937 \\
\hline
\end{tabular}

\section{Graph No -1}

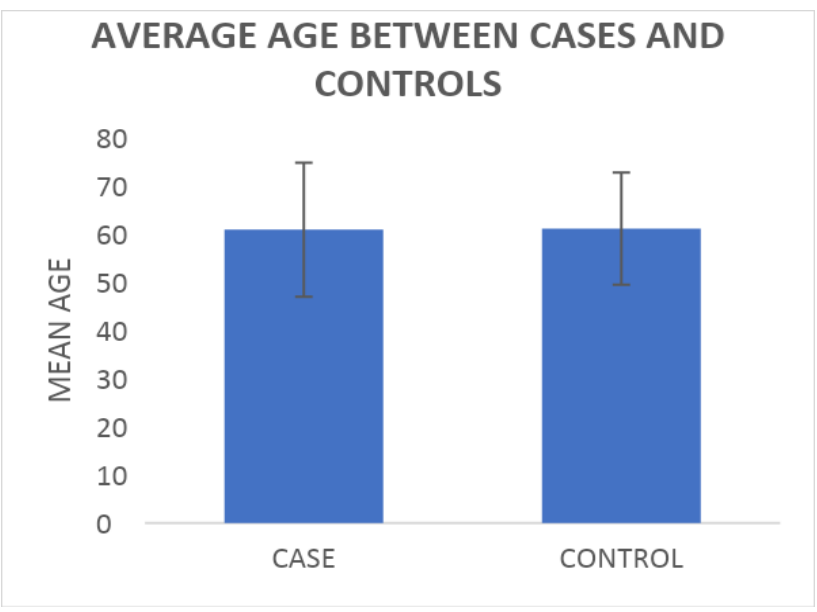

2-Mean Vitamin D Levels amongst Cases and Controls

In our study, we recorded that mean vitamin D levels in the case group were $15.46 \pm 8.41 \mathrm{ng} / \mathrm{ml}$ and the control group were $26.74 \pm 13.28 \mathrm{ng} / \mathrm{ml}$.

Table No -2

\begin{tabular}{|l|c|c|c|}
\hline Bio Chemical Parameters & Case $(\mathbf{n}=\mathbf{4 8})$ & Control $(\mathbf{n}=\mathbf{4 8})$ & P value \\
\hline Serum vitamin D $(\mathrm{ng} / \mathrm{dl})$ & $15.46 \pm 8.41$ & $26.74 \pm 13.28$ & $<0.001$ \\
\hline
\end{tabular}

\section{Graph No-2}

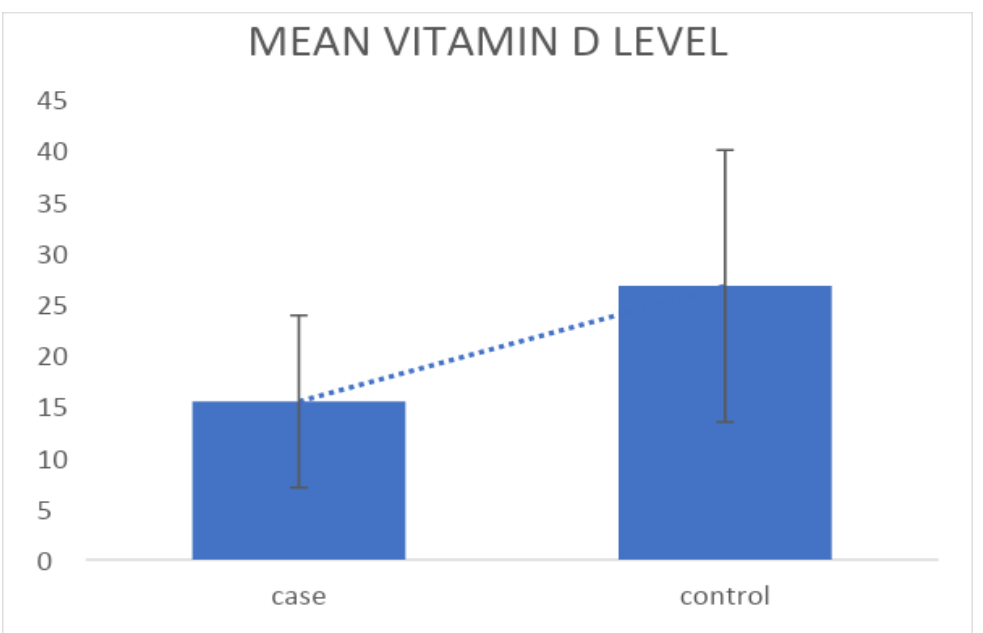

Distribution of Cases and Controls according to Vitamin D Deficiency Status (Cut Off $20 \mathrm{ng} / \mathrm{ml}$ ) Table No-3

\begin{tabular}{|l|c|c|}
\hline VIT D DEFICIENCY $(<20 \mathrm{ng} / \mathrm{ml})$ & CASE $(\mathrm{n}=48)$ & CONTROL $(\mathrm{n}=48)$ \\
\hline YES & 35 & 13 \\
\hline NO & 19 & 29 \\
\hline
\end{tabular}


Graph No-3

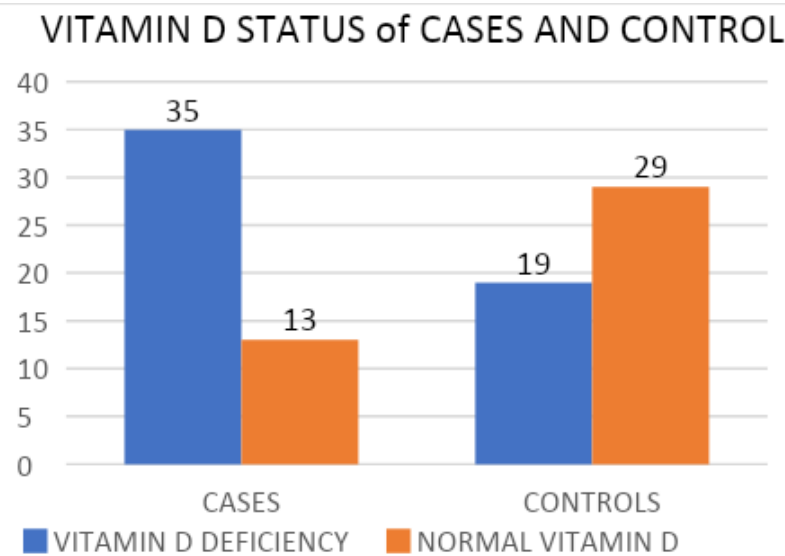

\section{Discussion}

The present study entitled "The study of 25hydroxy vitamin D level in ischaemic stroke patients" was carried out in the Post Graduate, KPS Institute of Medicine, G.S.V.M. Medical College, Kanpur from January 2019 to October 2020 to find out the role of vitamin D and its various correlation in cases of acute ischemic stroke patients.

\section{Age and Gender Variation with Stroke and Vitamin \\ D Levels}

In our study, out of 96 study subjects, 48 subjects were in the case group with $29(61 \%)$ males and 19 (39\%) females. Out of 48 subjects of the control group, 28 (42\%) were males and 20 (58\%) were females. The mean age of the case group was $60.95 \pm 13.9$ years which is comparable to the mean age of the control group which was $61.16 \pm$ 11.6 years. There was no statistically significant difference observed between the mean age of the two groups $[\mathrm{p}$ value $=0.937$ (not significant)]

\section{Vitamin D and Risk of Ischemic Stroke}

In the study, we recorded that mean vitamin D levels in the case group were $15.46 \pm 8.41 \mathrm{ng} / \mathrm{ml}$ and the control group were $26.74 \pm 13.28 \mathrm{ng} / \mathrm{ml}$. Thus, mean vitamin D levels in ischemic stroke patients were lower as compared to age and sexmatched controls. To find out whether this difference was statistically different we distributed the subjects in both groups in the contingency table according to their vitamin $\mathrm{D}$ deficiency status taking vitamin $\mathrm{D}<20 \mathrm{ng} / \mathrm{ml}$ as cut-off. On evaluating we found that $35(73 \%)$ of subjects with vitamin D deficiency had an ischemic stroke as compared to 19 (40\%) subjects with no stroke. Using chi-square, we got a p-value of 0.0009 which is statistically significant. The odds ratio of our study was 4.109 within a $95 \%$ confidence interval of 1.74 to 9.71 . This reveals that vitamin $\mathrm{D}$ levels are inversely related to the occurrence of ischemic stroke and levels less than $20 \mathrm{ng} / \mathrm{ml}$ increase the odds of an ischemic stroke four times as compared to age and sex-matched population with the confidence interval of $95 \%(p=0.0013)$.

Jaydip Ray Chaudhuri, et $\mathbf{a l}^{\mathbf{4}}$ (2014) recruited 250 consecutive ischemic stroke patients and 250 age and sex-matched, and found that 25hydroxyvitamin D deficiency had an independent association with ischemic stroke. Their analysis showed an independent association of 25hydroxyvitamin D deficiency with ischemic stroke $(\mathrm{P}<0.001$, odds ratio: 1.6 ; 95\% CI 1.2-2.8) which was similar to our study $(\mathrm{p}=0.0013$, odds ratio 4.117; 95\%; CI 1.73-9.71). Our study was also similar to the above study concerning study design.

Kenneth E. S. Poole, et al ${ }^{30}(\mathbf{2 0 1 5})$ compared the serum 25- dihydroxy vitamin D levels of 44 patients admitted with the first-ever stroke with results obtained by measuring 96 healthy ambulant elderly subjects every 2 months for 1 year. They concluded that Vitamin D is a potential risk marker for stroke. Their study supported our 
observation. Their study as well as the sample size was quite similar to ours.

Majumdar, et $\mathbf{a l}^{31}(\mathbf{2 0 1 5})$ cross-sectionally evaluated the relationship between vitamin D deficiency and stroke in the high-risk Asian Indian population in age- and gender-matched, 239 ischemic stroke patients and 241 control subjects and reported an inverse association between serum $25(\mathrm{OH}) \mathrm{D}$ concentration and risk of ischemic stroke. Subjects with severely low $25(\mathrm{OH})$ D levels $(<10 \mathrm{ng} / \mathrm{ml})$ were found to have 3.13-fold (95\% confidence interval (CI), (1.22-8.07)) increased risk of ischemic stroke as compared with those with higher levels. The cut-off value in their study for vitamin D levels was $<10 \mathrm{ng} / \mathrm{ml}$, while we used $<20 \mathrm{ng} / \mathrm{ml}$ based on the levels recommended by the Endocrine Society.

Fahmy, Sharaf H. et $\mathbf{a l}^{\mathbf{3 3}}$ (2019) had studied 48 acute ischemic stroke patients and 48 matched healthy control subjects. They found that stroke patients had significantly lower serum vitamin D levels compared to healthy subjects. An increased risk of stroke of 2.88 times was found in insufficient vitamin D in comparison to sufficient subgroup and this likelihood increases to be 13.78 times in the deficient compared to sufficient subgroups. They concluded that vitamin D deficiency increases the risk of acute ischemic stroke. Our study type and sample are similar to the above study and their results also support our findings.

Talebi, et $\mathbf{a l}^{32}$ (2020) according to the results of this study median phosphorus and vitamin D levels were significantly lower in stroke patients than the controls $(3.6[3.02-4.21]$ vs. 4.2 [3.84.6]) and (15.1 [8.2-27.9] vs. 22.7 [10.4-39.2]), respectively. Multiple logistic regression analysis showed that the ischemic stroke was positively associated with the vitamin D level, they concluded that lower serum levels of vitamin D3 were associated with ischemic stroke.

\section{Conclusion}

In our study, ischaemic stroke patients had significantly low levels of Vitamin D (mean vit D level = $15.46 \mathrm{ng} / \mathrm{ml})$. Vitamin D levels may be a potentially modifiable cerebrovascular risk factor.

\section{Bibliography}

1. Biswas M, Sen S, Simmons J. Etiology and risk factors of ischemicstroke in Indian American patients from a hospital-based registry in New Jersey USA. Neuro Asia 2009; 14:81-86.

2. World Health Organization. World Health Report 2002.Switzerland, Geneva: WHO, 2002

3. Wieberdink RG, Ikram MA, Hofman A, Koudstaal PJ, Breteler MM. Trends in stroke incidence rates and stroke risk factors in Rotterdam, the Netherlands from 1990 to 2008.Eur J Epidemiol. 2012; 27:287-295. doi: 10.1007/s10654-0129673-y.

4. Chaudhuri JR, Mridula KR, Alladi S, Umamahesh M, Balaraju B, Swath A, et al. Serum 25-hydroxyvitamin D deficiency in ischemic stroke and subtypes in Indian patients. J STROKE. 2014; 16:44.

5. Jemma C. HopewellRobert Clarke et al. Emerging Risk Factors for Stroke. Stroke. 2016; 47:1673-1678.

6. Zhou R, Wang M, Huang H, Li W, Hu Y, $\mathrm{Wu} \mathrm{T}$. Lower vitamin $\mathrm{D}$ status is associated with an increased risk of ischemic stroke: a systematic review and meta-analysis. Nutrients. (2018) 10: E277. DOI: $10.3390 /$ nu10030277.

7. Turetsky A, Goddeau RP, Henninger N. Low serum vitamin D is independently associated with larger lesion volumes after ischemicstroke. J Stroke Cerebrovasc Dis. (2015) 24:1555-63. DOI: 10.1016/j.jstrokecerebrovasdis.2015.03.05 1.

8. Huang H, Zheng T, Wang S, Wei L, Wang Q, Sun Z. Serum 25- hydroxyvitamin D predicts early recurrent stroke in ischemic stroke patients. NutrMetab Cardiovasc 
Dis. (2016) 26:908-14. DOI:

10.1016/j.numecd.2016.06.009.

9. Pasco JA, Henry MJ, Kotowicz MA, Sanders KM, Seeman E, Pasco JR, et al. Seasonal periodicity of serum vitamin $\mathrm{D}$ and parathyroid hormone, bone resorption and fractures: the Geelong Osteoporosis Study. J Bone Miner Res 2004; 19:752758. PMID: 15068498

10. Holick MF. Vitamin D deficiency. N Engl J Med 2007;357:266-281. PMID: 17634462.

11. Feldman D, Pike JW, Glorieux F. Vitamin D. 2nd ed. Philadelphia, PA: Elsevier (2005).

12. Van Schoor, N.M.; Lips, P. Worldwide Vitamin D Status. Best Pract. Res. Clin. Endocrinol. Metab. 2011, 25, 671-680.

13. Mithal, A.; Wahl, D.A.; Bonjour, J.P.; Burckhardt, P.; Dawson-Hughes, B.; Eisman, J.A.; El-Hajj Fuleihan, G.; Josse, R.G.; Lips, P.;Morales-Torres, J.; et al. Global vitamin D status and determinants of hypovitaminosis D. Osteoporos Int. 2009, 20, 1807-1820.

14. Van der Meer, I.M.; Middelkoop, B.J.; Boeke, A.J.; Lips, P. Prevalence of vitamin D deficiency among Turkish, Moroccan, Indian and sub-Sahara African populations in Europe and their countries of origin: An overview. Osteoporos. Int. 2011, 22, 1009-1021.

15. Afzal S, Nordestgaard BG. Vitamin D, hypertension and ischemic stroke in 116 655 individuals from the general population: a genetic study. Hypertension. (2017)

doi:10.1161/HYPERTENSIONAHA 117.09411 .

16. Pittas AG, Harris SS, Stark PC, DawsonHughes B. The effects of calcium and vitamin D supplementation on blood glucose and markers of inflammation in nondiabetic adults. Diabetes Care. (2007) 30:980-6. DOI: 10.2337/dc06-1994.
17. Feng C, Tang N, Huang H, Zhang G, Qi $\mathrm{X}$, Shi F. 25-Hydroxy vitamin $\mathrm{D}$ level is associated with total MRI burden of cerebral small vessel disease in ischemic stroke patients. Int J Neurosci. (2019) 129:49-54. DOI: 10.1080/00207454.2018.1503182.

18. Wang Y, Ji H, Tong Y, Zhang ZB. Prognostic value of serum 25hydroxyvitamin D in patients with stroke. Neurochem Res. (2014) 39:1332-7. DOI: 10.1007/s11064-014-1316-0.

19. Makariou SE, Michel P, Tzoufi MS, Challa A and Milionis HJ: Vitamin D and stroke: promise for prevention and a better outcome. CurrVascPharmacol. 2014 Jan; 12(1):117-24.

20. Parker GB, Brotchie H, Graham RK. Vitamin D and depression. J Affect Disord. (2017) 208:56-61. DOI: 10.1016/j.jad.2016.08.08233. Borgi L, McMullan C, Wohlhueter A, Curhan GC, Fisher ND, Forman JP. Effect of Vitamin $\mathrm{D}$ on endothelial function: a randomized, double-blind, placebo-controlled trial. Am J Hypertension 2017; 30:124-129. DOI: 10.1093/ajh/hpw135.

21. Kienreich K, Grubler M, Tomaschitz A, Schmid J, Verheyen N, Rutters F et al. Vitamin D, arterial hypertension \& cerebrovascular disease. Indian J Med Res 2013; 137: 669-679.

22. Thapa L, Pokhrel B, Shrestha A, Pradhan M, Bhandari TR, Shrestha S, et al. Status of vitamin $\mathrm{D}$ and its association with stroke risk factors in patients with acute ischemic stroke in a tertiary care hospital. J Nepal Med Assoc. 2014; 52:935.

23. Gupta A, Prabhakar S, Modi M, Bhadada SK, Lal V, Khurana D: Vitamin D status and risk of ischemic stroke in North Indian patients. Indian J Endocrinol Metab. 2014 Sep; 18(5):721-5.

24. Ford L, Graham V, Wall A, Berg J. Vitamin D concentrations in a UK inner- 
city multicultural outpatient population. Ann Clin Biochem 2006; 43: 468-473.

25. Hawkins R. Total 25-OH vitamin D concentrations in Chinese, Malays and Indians. Ann Lab Med 2013; 33: 156-158.

26. Sun Q, Pan A, Hu FB, Manson JE, Rexrode KM. 25-Hydroxyvitamin D levels and the risk of stroke: a prospective study and metaanalysis. Stroke 2012; 43: 14701477.

27. Brøndum-Jacobsen P, Nordestgaard BG, Schnohr P, Benn M. 25- hydroxyvitamin $\mathrm{D}$ and symptomatic ischemic stroke: an original study and meta-analysis. Ann Neuro 2013; 73: 38-47.

28. Michos ED, Reis JP, Post WS, Lutsey PL, Gottesman RF, Mosley TH et al. 25(OH)D deficiency is associated with fatal stroke among whites but not blacks: the NHANES-III linked mortality files. Nutrition 2012; 28: 367-371.

29. Robinson-Cohen C, Hoofnagle AN, Ix JH, Sachs MC, Tracy RP, Siscovick DS et al. Racial differences in the association of serum 25- hydroxyvitamin D concentration with coronary heart disease events. JAMA 2013; 310: 179-188.

30. Kenneth E. S. Poole, Nigel Loveridge, Peter J. Barker, David J. Halsall, Collette Rose, Jonathan Reeve and Elizabeth A. Warburton; Reduced Vitamin D in Acute Stroke; stroke AHA journal.

31. Majumdar V, Prabhakar P, Kulkarni GB, Christopher R: Vitamin D status, hypertension and ischemic stroke: a clinical perspective. J Hum Hypertension. 2015 Nov; 29(11):669-74.

32. Abolfazl Talebi, Alireza Amirabadizadeh, Samaneh Nakhaee, Zahra madi and Seyed Mohammad Mousavi-Mirzaei: Cerebrovascular disease: how serum phosphorus, vitamin D and uric acid levels contribute to the ischemic stroke; BMC Neurology (2020) 20:116
33. Fahmy, E., Sharaf, S., Helmy, H. et al. Vitamin D status in acute ischemic stroke: relation to initial severity and short-term outcome.Egypt J Neuro-Psychiatry Neurosurgery 55, 18 (2019). 